\title{
Fluidic Assisted Thin-Film Device Heterogeneous Integration: Surface Tension as Driving Force and Magnetic as Guiding Force
}

\author{
Jing Xiao ${ }^{1}$, Ritesh Ray Chaudhuri ${ }^{2}$ and Sang-Woo Seo ${ }^{2}$ \\ ${ }^{1}$ College of Electrical and Information Engineering, Hunan University, Changsha, Hunan, 410082, P.R. China \\ ${ }^{2}$ Department of Electrical Engineering, The City College of New York, 160 Convent Avenue, New York, NY 10031, USA \\ Email:swseo@ccny.cuny.edu
}

\begin{abstract}
This paper demonstrates a fluidic assisted heterogeneous integration of optical thin-film device using surface tension as driving force and magnetic field as guiding force. Thinfilm devices can be auto-aligned and integrated using surface tension onto their predesigned locations on a host substrate due to minimization of interfacial energy. By inserting a layer of nickel $(\mathrm{Ni})$ into device metallization step, magnetic force was employed to increase mobility and contact probability of thin-film devices to their binding sites on the host substrate. A thin-film gallium arsenide (GaAs) inverted MetalSemiconductor-Metal (MSM) photodetector (PD) has been successfully integrated onto a silicon host substrate with the proposed integration approach. The influence of the nickel layer to the PD performance was also investigated. Due to the self-assembly capability and thin-film format of the device, the proposed method has potential for wafer-scale implementation and is compatible with the matured silicon-based CMOS technology. This is a critical step towards a scalable manufacturing process to create advanced photonic/optoelectronic systems that are low-cost, compact, high performance, and complex in multi-material functionality.
\end{abstract}

Key words:

Thin-film heterogeneous integration, Fluidic self-assembly, Surface tension, Magnetic force

\section{Introduction}

In the early 1960s, the appearance of semiconductor laser has promoted the development of integrated optics [1, 2]. Since then, researchers began to fabricate the photonic/optoelectronic integrated circuits (ICs) [3, 4] using various materials and different manufacturing processes in order to achieve advanced microsystems. Monolithic integration technology, matured in the electronic ICs, is still facing difficulty to integrate different photonic devices in a common substrate with both high performance and low cost implementations. A diaphragm transfer integration method [5-8] has been reported to combine different thin-film devices, heterogeneously, onto a single host substrate. This approach allows the devices for integration to be independently grown and fabricated on their preferred growth substrates and selectively post-integrate them in a thin-film format onto a final host substrate. While wafer/chip-towafer bonding integration approaches [9-11], either the direct bonding or adhesive bonding techniques, uses a whole wafer/chip resulting in excess material wastage after device definition using etching, the diaphragm transfer method can fabricate the devices in array to maximize the expensive III-V material usage. In recent publications [12, 13], this integration method is further developed by combining self-assembly approach, which uses surface tension as the driving force to auto-align and integrate thin-film device on predefined integration locations of a host substrate.

In this paper, we introduce magnetic force as an efficient guiding mechanism of thin-film devices in aqueous environment. Due to the thin-film format of the devices, conventional mechanical fluidic agitation methods $[14,15]$ used for self-assembly process are not efficient to move the thin-film devices in aqueous environment. Because the interacting force between the thin-film devices and a host substrate is much stronger compared to the case with thicker devices with a gravity factor [16]. By using a thin layer of magnetic material in the device metallization step, magnetic force has been employed to improve the mobility of the thin-film devices and as a result increasing the 
probability of contact between the devices and integration locations in a host substrate. The demonstration will be a critical step for parallel wafer-scale thin-film device integration.

\section{Surface-tension driven heterogeneous integration}

The surface-tension driven heterogeneous integration, including the thin-film device fabrication, host substrate preparation, and the integration process [12,13], is summarized in Fig. 1. The GaAs based photonic devices (here, MSM PDs) can be grown, fabricated, and optimized on their preferred growth substrate as shown in Fig. 1(a). The top pattering (Fig. 1(a1-a3)) and metal definition (Fig. 1(a4-a5)) are done by a dual-layer lift-off process (here, SF11 and AZ 5214 photoresists are employed) and thermal evaporation. Then, the thin-film PDs are formed by separation from or removal of the growth substrate (Fig. 1(a7-a8)). Finally, the devices, embedded in and protected by the Apiezon W wax, are bonded to a Mylar diaphragm, which is stretched across and bonded onto a silicon ring. Later, the wax is dissolved with trichloroethylene (TCE). Fig.1 (a9) and (a10) shows the top view and bottom view of the arrayed thin-film PDs on the diaphragm demonstrating maximal material usage especially for sparse array of optoelectronic device integration. The host substrate is separately prepared on a silicon dioxide coated silicon wafer and electrical contacts were realized evaporating chromium and gold (Cr\Au thickness: $15 \mathrm{~nm} \backslash 145 \mathrm{~nm}$ ). After the fabrication of electrical pads , using micro-contact printing method with PDMS stamp [13], self-assembled monolayer (SAM) can be selectively formed at the binding sites (Fig.1 (b)). This process changes the integration locations into hydrophobic while keeping other areas hydrophilic. Then, the substrate is passed through a lubricant-water interface to form attracting medium (lubricant) for the integration on the specific SAM coated gold surfaces in water Fig.1 (c2). The lubricant used in the process is an evaporable solvent, OptiClear S2. The integration starts from dispensing of thin-film devices towards the integration areas. Once the devices interact with the binding sites through the attracting medium, they are automatically aligned and integrated on the host substrate due to free energy minimization in water. After the completion of integration, the lubricant can be evaporated and permanent electrical contact between the device and integration pads can be achieved by direct metal-to-metal bonding.

\section{Magnetic guiding force for the integration}

\subsection{Magnetic as the guiding force}

In order to further develop the surface-tension driven heterogeneous integration method and improve the efficiency of the self-assembly process, assistive magnetic force, by deposition of a thin nickel layer into the thin-film device metallization step, has been employed in this work to guide the thin-film devices onto the integration locations of the host substrate. While unbounded thin-film devices can be moved easily and fill vacancies of the binding sites, the bounded devices with good alignment will be held in place due to the lubricant, and misaligned devices will be fitted onto the desired locations.

The experimental setup for the integration with magnetic assistive guiding force is shown in Fig.2. A N52+ Grade Neodymium Ferro Boride (NdFeB) block having a dimension of 1/2 inch $\times 1 / 2$ inch and a pull of 35 pounds was used as the magnetic block. The value of the magnetic field along the axis of magnetization can be calculated as [17]:

$$
B_{x}=\frac{B_{r}}{\pi}\left(\arctan \left(\frac{l w}{2 x \sqrt{\left(4 x^{2}+l^{2}+w^{2}\right)}}\right)-\arctan \left(\frac{l w}{2(t+x) \sqrt{4(t+x)^{2}+l^{2}+w^{2}}}\right)\right)
$$

where $B_{x}$ is the magnetic field along the axis of magnetization. $l, w$ and $t$ are the length, width and thickness of the magnet respectively. $x$ is the distance from the surface of the magnet along the axis of magnetization. $B_{r}$ is the residual flux density and for $\mathrm{NdFeB}$ grade $\mathrm{N} 52$ magnets, the value of $B_{r}$ is estimated to be 14300 Gauss. The magnetic force can be controlled by using magnets of different size and combinations. The thin-film devices were introduced on the host substrate with the help of a transfer diaphragm. As the integration is carried out keeping the host substrate submerged in water, the thin-film devices are generally randomly distributed on it. The movement of the magnetic block underneath the Petri dish causes the thin-film devices to move on the host substrate and subsequently guides them onto the desired binding sites. The distance between the magnetic block and Petri dish can be roughly controlled by adjusting the height of the Petri dish holder, and here the distance was kept to be around $0.7 \mathrm{~mm}$. Considering the thickness of the glass petri dish and host substrate to be $1.88 \mathrm{~mm}$ and $500 \mu \mathrm{m}$ respectively, the resulting magnetic field on the top surface of silicon wafer for the $0.7 \mathrm{~mm}$ gap can be calculated around 3700 Gauss. This distance can be smaller and more accurate by employment of micro positioning stages. The camera in the setup is used to record the movement of the thin-film devices and the surface-tension driven 
integration process. Fig. 3 shows that thin-film devices can be guided to any desired location with the help of magnetic force. It was verified from the experiments that Ni thickness of $100 \mathrm{~nm}$ in the device layer is effective and efficient in moving the thin film devices. Lower thickness of $\mathrm{Ni}$ can be investigated to reduce the influence on the devices performance while retaining the capability to move the thin-film devices freely.

\subsection{Influence of magnetic material (Ni) on device performance}

The influence of the Ni layer to PD performance was first investigated by measuring the dark current of the PDs with different thickness of evaporated Ni. GaAs wafer, with structure of GaAs/AlAs/ GaAs stack (1/0.25/350 $\mu \mathrm{m})$, was used to test the influence. Three samples (one without $\mathrm{Ni}$ and the other two with different Ni layer thicknesses) were fabricated: Cr\Au layer with thickness of $15 \backslash 145 \mathrm{~nm}$, Cr\Ni\Au with $15 \backslash 100 \backslash 145 \mathrm{~nm}$, and Cr\Ni\Au with $15 \backslash 150 \backslash 145 \mathrm{~nm}$. After metallization of the PDs, the samples were placed in an oven for the annealing process. The metal-semiconductor Schottky contact can be improved by thermal annealing at a certain high temperature after the metal deposition $[18,19]$. As shown in Fig. 4, the optimal annealing temperature is around $200^{\circ} \mathrm{C}$ without nickel and $190^{\circ} \mathrm{C}$ with nickel. The influence of nickel on the PD performance is shown in Fig. 5. It is true that the additional magnetic metal layer can degrade the PDs' performance. For instance, at a Ni thickness of $100 \mathrm{~nm}$, the dark current is roughly two times the dark current when there is no Ni layer. However, this is acceptable since the influence is not significant and $100 \mathrm{~nm}$ of evaporated $\mathrm{Ni}$ is sufficient to apply the assistive force necessary to move the thin-film device on the host substrate as previously demonstrated.

\section{Fluidic assisted thin-film heterogeneous integration}

During the integration process, the surface-tension using a lubricant is employed to integrate, self-align, and hold the thin-film devices on the binding sites of a host substrate. On the other hand, the magnetic force is employed as the assistive force to move the devices and guide them onto the binding sites on the host substrate in order to improve the integration efficiency. Fig. 6 demonstrates the role of magnetic force in guiding a single thin-film device onto the binding sites for integration. After the device is attracted by the lubricant, it is held by surface tension and remained fixed at the binding sites. Due to the fact that surface-tension at the micro scale is much stronger than the magnetic force, the unbounded thinfilm devices could be easily removed from the host substrate by magnetic force, while the bounded device would remain on the binding sites, ready for further processing. Fig.6 (a-c) clearly shows that the flipping of the thin-film device can be performed by changing the direction of the magnetic force. After guiding the thin-film device towards the integration sites and aligning it roughly parallel to the integration pads, the thin-film device was brought in contact with the lubricant on both of the binding sites and self-aligned in the location due to the minimization of interfacial energy as represented in Fig. 6(d-h). Fig. 6(e) shows that one of the binding sites has been attracted by lubricant but not the other one. Through the rotation of the magnetic block, the other side of the device also contacted to the attracting medium and self-aligned onto the ideal integration location, which represents the global minimal energy state. The final alignment is related to the quality of the binding sites of the thin-film device and the substrate, as well as the lubricant amount and its formation. Fig. 6 (h) shows a GaAs thin-film inverted-MSM PD was successfully integrated onto the binding sites of the silicon host substrate. The thin-film GaAs MSM PD structure used for the magnetic assisted fluidic self-assembly was fabricated using the same process of Fig .1(a1-a10). The GaAs epi wafer has the structure of GaAs device layer/AlAs etching stop layer/ GaAs growth substrate layer with thickness of $1 \mu \mathrm{m} / 0.25 \mu \mathrm{m} / 350 \mu \mathrm{m}$. Top pattering and metal deposition with $\mathrm{Cr} l \mathrm{Ni} \backslash \mathrm{Au}$ $(15 \mathrm{~nm} \backslash 100 \mathrm{~nm} \backslash 145 \mathrm{~nm})$ were done by a dual-layer lift-off process as shown in Fig. 1(a1-a5). The interdigitated fingers are $70 \mu \mathrm{m}$ length, $3 \mu \mathrm{m}$ width, and $6 \mu \mathrm{m}$ finger spacing. The photo-active region is $60 \mu \mathrm{m} \times 50 \mu \mathrm{m}$ and the dimension of the bonding pads is $80 \mu \mathrm{m} \times 45 \mu \mathrm{m}$, as shown in Fig. 1(c1). Citric acid: hydrogen peroxide (4:1) was employed for the mesa definition, and the devices were etched down to the AlAs stop layer (Fig. 1(a6)). The PD's dimension is $200 \mu \mathrm{m} x$ $100 \mu \mathrm{m}$.Then, the devices were embedded in Apiezon W wax and AlAs layer was selectively removed using $10 \%$ hydrogen fluoride (HF) solution to achieve the device separation from the growth substrate as shown in Fig. 1(a7-a8). The final thickness of the PDs is $\sim 1 \mu \mathrm{m}$.

After the integration water was carefully drained out and the host substrate was placed in an oven at $190^{\circ} \mathrm{C}$ to evaporate the lubricant and provide stable metal-to-metal contact between the thin-film PD and the host substrate (Fig. 7). And, the electrical contact was confirmed by the photocurrent measurement of integrated PD, as shown in Fig. 7, using a Keithley 2400 source measurement unit.

The success rate of the proposed integration approach can be expressed in terms of the number of devices integrated with respect to the number of devices dispensed into the fluidic environment for a specific attempt. As the success rate mainly depends on the guiding magnetic force and surface tension, precise control of the parameters related to them can greatly improve the yield. The guiding magnetic force can be controlled using a multi-axis translation stage for efficient device 
movement in fluidic environment. The design and geometry of the binding sites on the host substrate optimizes the integration force and improve the alignment accuracy by controlling the volume of the attracting medium. In some cases, the devices flipped and got attached to the binding sites with the metal side facing up (Fig. 8). As the semiconductor material, GaAs, is slightly hydrophobic [20,21], the backside of the device was attracted and held by the lubricant. This could be overcome by surface treatment of the backside to modify its wetting property from hydrophobic to hydrophilic, for instance, by depositing a few nanometers of $\mathrm{SiO}_{2}$ or $\mathrm{TiO}_{2}$ during thin-film device fabrication process. Then, the misaligned device (wrong side of thin-film device) can be moved away by the assistive magnetic force and thus releasing the integrated pads to be used for correct device integrations. In addition, the contact probability could be further increased by directing the devices' immersion in the water bath to bring the metal-side facing the substrate. This could probably be done by transfer diaphragm with embedded devices in a water-soluble polymer, like poly-acrylic acid (PAA).

\section{Conclusions}

This work demonstrates that magnetic guiding force can be employed to develop the surface-tension driven thin-film device heterogeneous integration process more effectively and efficiently. The magnetic force improves the mobility of thin-film devices and increases the contact probability of thin-film devices to the binding sites on a host substrate. To implement the magnetic guiding force, thin-film devices have a thin layer of $\mathrm{Ni}$ in their metallization layers. Experimentally, the addition of Ni in MSM photodetector structure does not significantly degrade the device performance. Effective movement and integration of a thin-film device on a foreign substrate in water have been demonstrated. The proposed integration approach provides automatic alignment of thin-film devices and potentially provides the waferscale/parallel integration capability. Based on thin-film format, photonic devices can be integrated on a foreign substrate using the proposed approach to develop multi-material, integrated photonic systems. As a future work, the integration of multiple devices on multiple integration sites will be studied to analyse the yield of this integration process. It will have potential applications in optical interconnection, optical signal processing systems, and bio/chemical sensors due to the ability of the proposed approach to integrate different materials and functions, including the electronics, photonics, microfluidics, RF, and MEMS.

\section{Acknowledgments}

This work was supported by the NSF grant (EEC-0823793), by the PSC-CUNY (60051-38 39) from USA and by the fundamental research funds for central universities from Hunan University, and by the National Natural Science Foundation of China (61404047).

\section{References}

[1] S. E. Miller, "Integrated Optics: An Introduction," Bell System Technical Journal, vol. 48, pp. 2059-2069, 1969.

[2] I. P. Kaminow, "Optical Integrated Circuits: A Personal Perspective," Lightwave Technology, Journal of, vol. 26, pp. 9941004, 2008.

[3] M. Yust, N. Bar - Chaim, S. H. Izadpanah, S. Margalit, I. Ury, D. Wilt, et al., "A monolithically integrated optical repeater," Applied Physics Letters, vol. 35, pp. 795-797, 1979.

[4] R. F. Leheny, R. E. Nahory, M. A. Pollack, A. A. Ballman, E. D. Beebe, J. C. DeWinter, et al., "Integrated In ${ }_{0.53} \mathrm{Ga}_{0.47} \mathrm{As} \mathrm{p}^{-1-n}$ f.e.t. photoreceiver," Electronics Letters, 16(10), 353-355, 1980.

[5] N. M. Jokerst, M. A. Brooke, S. Y. Cho, S. Wilkinson, M. Vrazel, S. Fike, et al., "The heterogeneous integration of optical interconnections into integrated microsystems," Ieee Journal of Selected Topics in Quantum Electronics, vol. 9, pp. 350-360, Mar-Apr 2003.

[6] S. W. Seo, S. Y. Cho, and N. M. Jokerst, "A thin-film laser, polymer waveguide, and thin-film photodetector cointegrated onto a silicon substrate," Ieee Photonics Technology Letters, vol. 17, pp. 2197-2199, Oct 2005.

[7] S. Dhar, A. Degiron, D. R. Smith, and N. M. Jokerst, "Planar Integrated Optical Detection of a Hybrid Long-Range Surface Plasmon Using an InGaAs Inverted-MSM Detector Bonded to Silicon," Ieee Photonics Technology Letters, vol. 22, pp. 841843 , Jun 2010.

[8] L. Luan, M. W. Royal, R. Evans, R. B. Fair, and N. M. Jokerst, "Chip Scale Optical Microresonator Sensors Integrated With Embedded Thin Film Photodetectors on Electrowetting Digital Microfluidics Platforms," Ieee Sensors Journal, vol. 12, p. 7, Jun 2012.

[9] D. Liang, J. E. Bowers, D. C. Oakley, A. Napoleone, D. C. Chapman, C. L. Chen, et al., "High-Quality 150 mm InP-to-Silicon Epitaxial Transfer for Silicon Photonic Integrated Circuits," Electrochemical and Solid State Letters, vol. 12, pp. H101-H104, 20092009.

[10] G. Roelkens, L. Liu, D. Liang, R. Jones, A. Fang, B. Koch, et al., "III-V/silicon photonics for on-chip and inter-chip optical interconnects," Laser \& Photonics Reviews, vol. 4, pp. 751-779, Nov 2010. 
[11] P. Wu, J. Liao, and Z. R. Huang, "Integration of inverted InGaAs MSM array on Si substrate through low temperature wafer bonding," Electronics Letters, vol. 48, pp. 38-39, Jan 52012.

[12] J. Xiao, F. C. Song, and S. W. Seo, "Fluidic Assisted Heterogeneous Integration of Photonic Thin-Film Devices," Ieee Photonics Technology Letters, vol. 22, pp. 1622-1624, Nov 2010.

[13] J. Xiao, F. C. Song, and S. W. Seo, "Surface-Tension Driven Heterogeneous Integration of Thin Film Photonic Devices Using Micro-Contact Printing for Multi-Material Photonic Integrated Circuits," Journal of Lightwave Technology, vol. 29, pp. 15781582, May 2011.

[14] H. O. Jacobs, A. R. Tao, A. Schwartz, D. H. Gracias, and G. M. Whitesides, "Fabrication of a cylindrical display by patterned assembly," Science, vol. 296, pp. 323-325, Apr 2002.

[15] X. R. Xiong, Y. Hanein, J. D. Fang, Y. B. Wang, W. H. Wang, D. T. Schwartz, et al., "Controlled multibatch self-assembly of microdevices," Journal of Microelectromechanical Systems, vol. 12, pp. 117-127, Apr 2003.

[16] U. Srinivasan, D. Liepmann, and R. T. Howe, "Microstructure to substrate self-assembly using capillary forces," Journal of Microelectromechanical Systems, vol. 10, pp. 17-24, Mar 2001.

[17] A. Tkachenko, R. F. Karlicek, and J. J. Q. Lu, "Directed self-assembly of mesoscopic dies using magnetic force and shape recognition," Electronic Components and Technology Conference (ECTC), 2014 IEEE 64th, pp. 2207-2212, 2014.

[18] P. Machac, "Thermal annealing of AuPt Schottky contacts on GaAs and AlGaAs," in Advanced Semiconductor Devices and Microsystems, 2000. ASDAM 2000. The Third International EuroConference on, 2000, pp. 143-146.

[19] L. C. Yang, R. X. Wang, S. J. Xu, Z. Xing, Y. M. Fan, X. S. Shi, et al., "Effects of annealing temperature on the characteristics of Ga-doped ZnO film metal-semiconductor-metal ultraviolet photodetectors," Journal of Applied Physics, vol. 113, Feb 2013.

[20] A. Gocalinska, K. Gradkowski, V. Dimastrodonato, L. O. Mereni, G. Juska, G. Huyet, et al., "Wettability and "petal effect" of GaAs native oxides," Journal of Applied Physics, vol. 110, Aug 2011.

[21] Y. C. Zhou, Z. H. Zhu, D. Crouse, and Y. H. Lo, "Electrical properties of wafer-bonded GaAs/Si heterojunctions," Applied Physics Letters, vol. 73, pp. 2337-2339, Oct 1998. 


\section{Figure caption}

Figure 1. (a) Thin-film device fabrication. (a1)-(a5) Dual-layer lift-off process: (a1) GaAs wafer with photoresists, (a2) AZ 5214 exposure, (a3) SF11 exposure, (a4) Metal deposition by thermal evaporation, (a5) After lift-off; (a6) mesa definition and etching, (a7) devices embedded in Apiezon W wax, (a8) Selective removal of sacrifice layer AlAs, (a9-a10) Fabricated PDs on Mylar diaphragm: (a9) front-side view, (a10) back-side view. (b) Selective SAM formation on the host substrate with micro-contact printing method: (b1) $\mathrm{SiO}_{2} / \mathrm{Si}$ wafer, (b2) Metallization, (b3) $\mathrm{O}_{2}$ plasma treatment, (b4) ODT on stamp, (b5) Micro-contact printing. (c) Fluidic assisted heterogeneous integration: thin-film PDs were dispensed and directed onto substrate followed by self-alignment to the binding sites due to free energy minimization; (c1) A thin-film PD with dimension labels, (c2) Host substrate with the selectively formed lubricant on the binding sites.

Figure 2. Experimental setup for the proposed integration with magnetic guiding force.

Figure 3. Movement of thin-film device on host substrate by magnetic guiding force. Arrow presents the direction of next movement. Device: GaAs $(\sim 1 \mu \mathrm{m})$ with metal CrlNi\Au 15\100\50nm; Substrate: $\mathrm{SiO}_{2}(6 \mu \mathrm{m}) / \mathrm{Si}$.

Figure 4. PD performance as a function of annealing temperature. (a) PD with Cr\Au (15\145nm) metallization, (b) PD with Cr\Ni\Au (15\100\145nm) metallization.

Figure 5. PD performance as a function of Ni thickness.

Figure 6. Fluidic assisted thin-film heterogeneous integration: surface-tension as driving force and magnetic as assistive guiding force.

Figure 7. Final structure of the thin-film GaAs PD integrated with silicon host substrate after the evaporation of lubricant, and the photocurrent measurement of the PD.

Figure 8. A misaligned GaAs PD with the metal-side facing up. 
Figure 1.

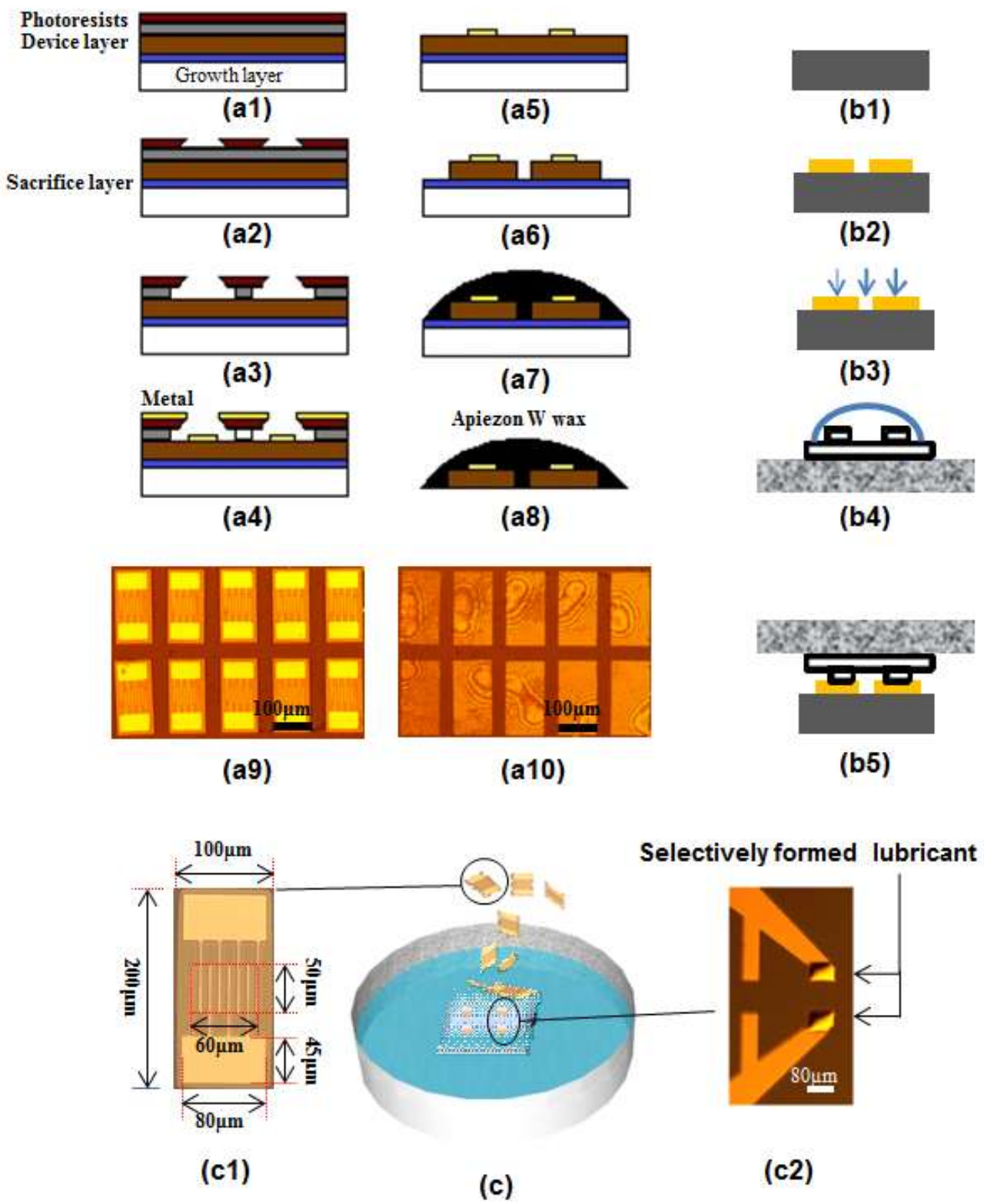


Figure 2.

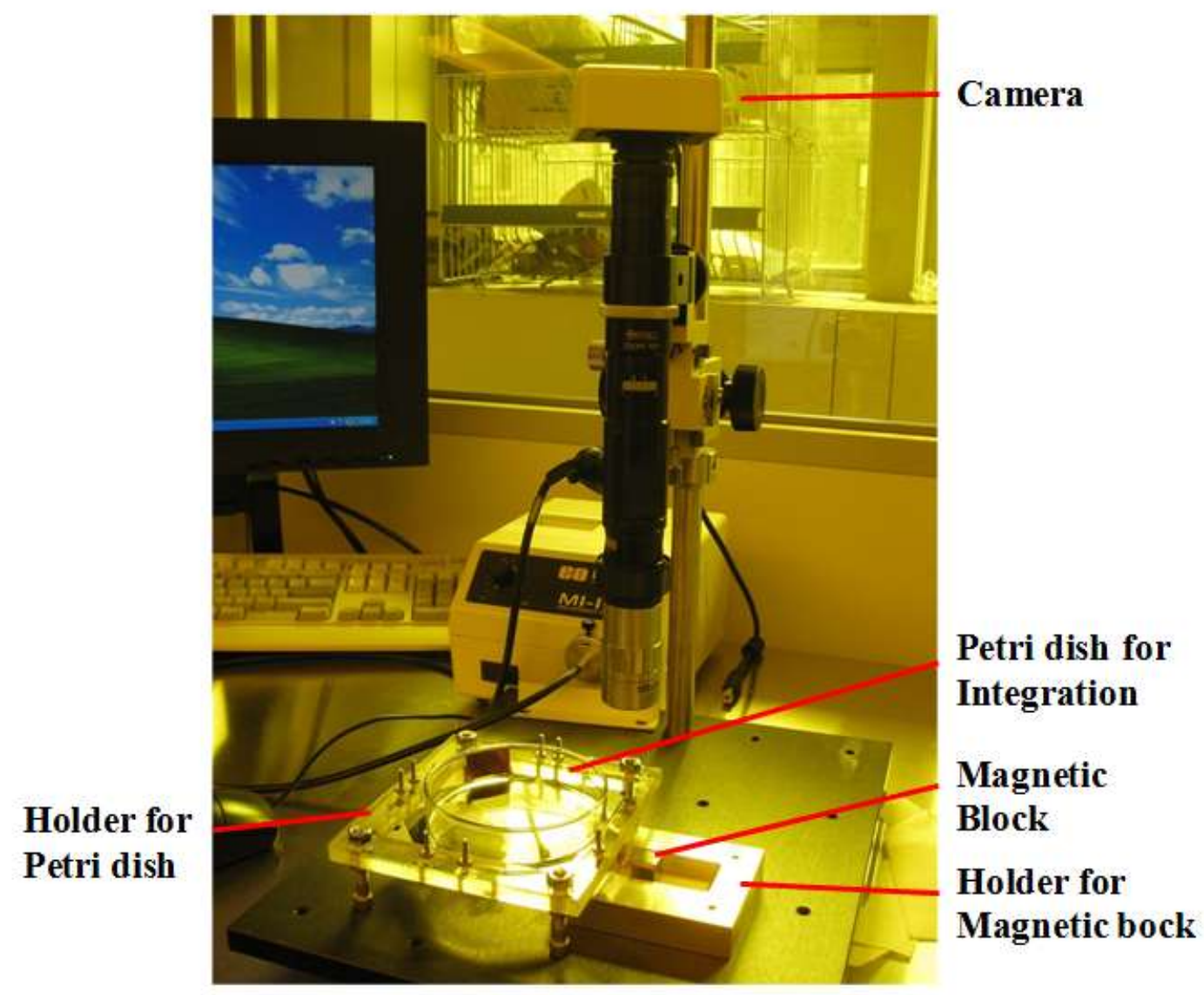


Figure 3.

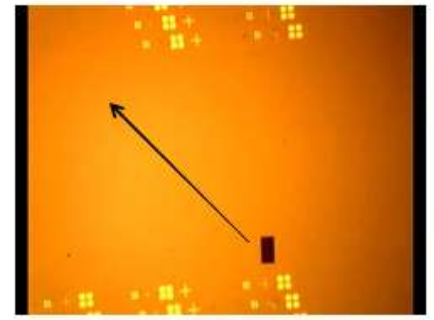

(a)

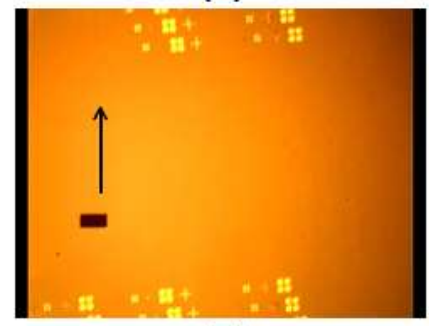

(c)

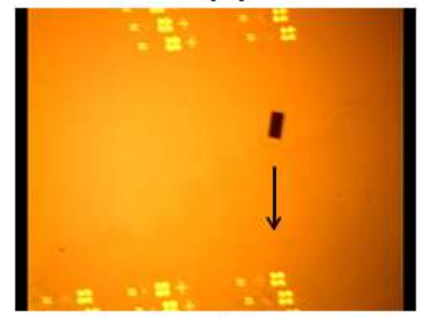

(e)

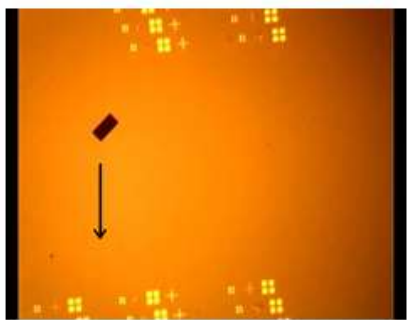

(b)

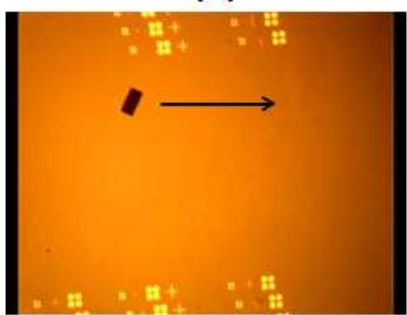

(d)

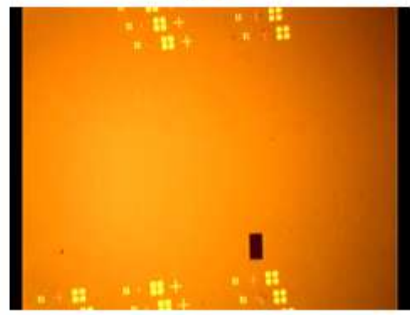

(f)

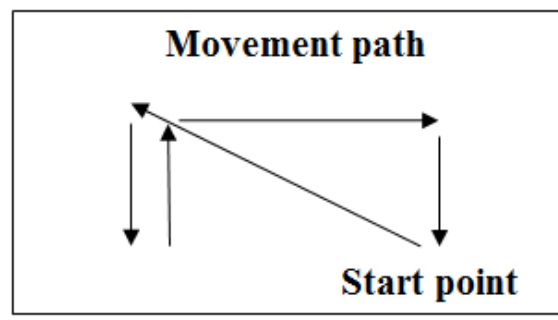

(g) 
Figure 4.

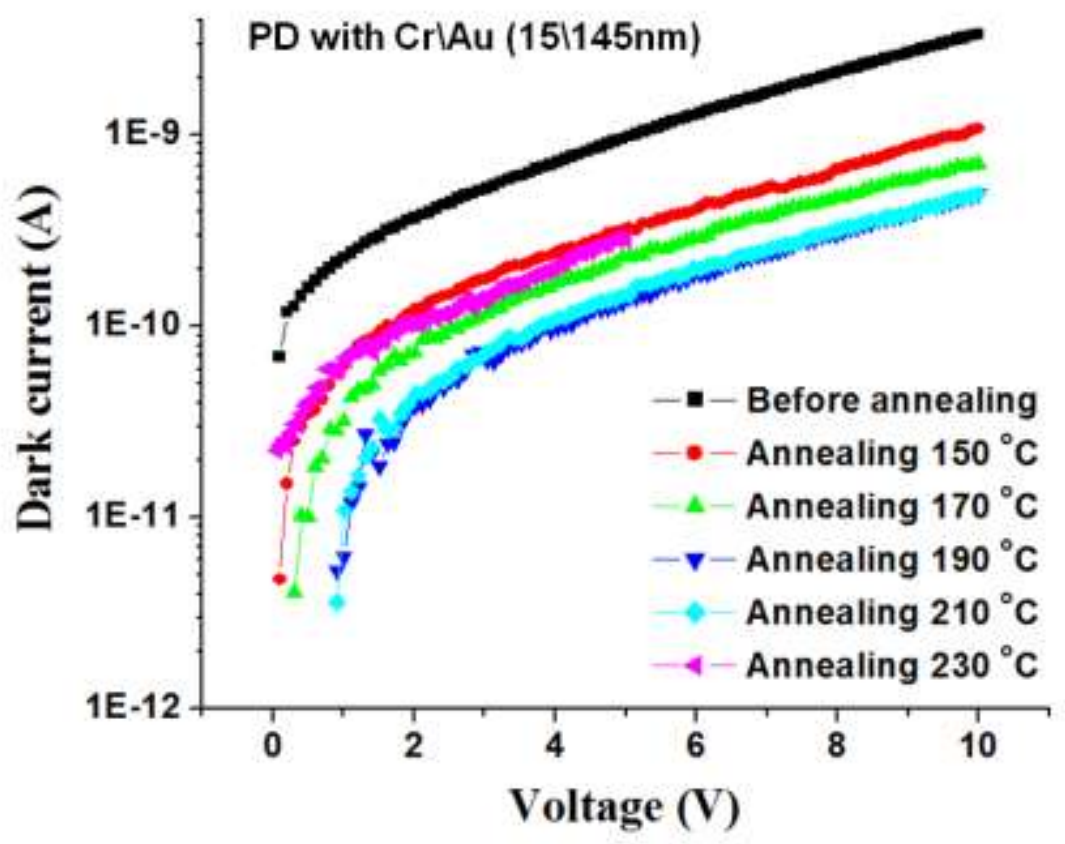

(a)

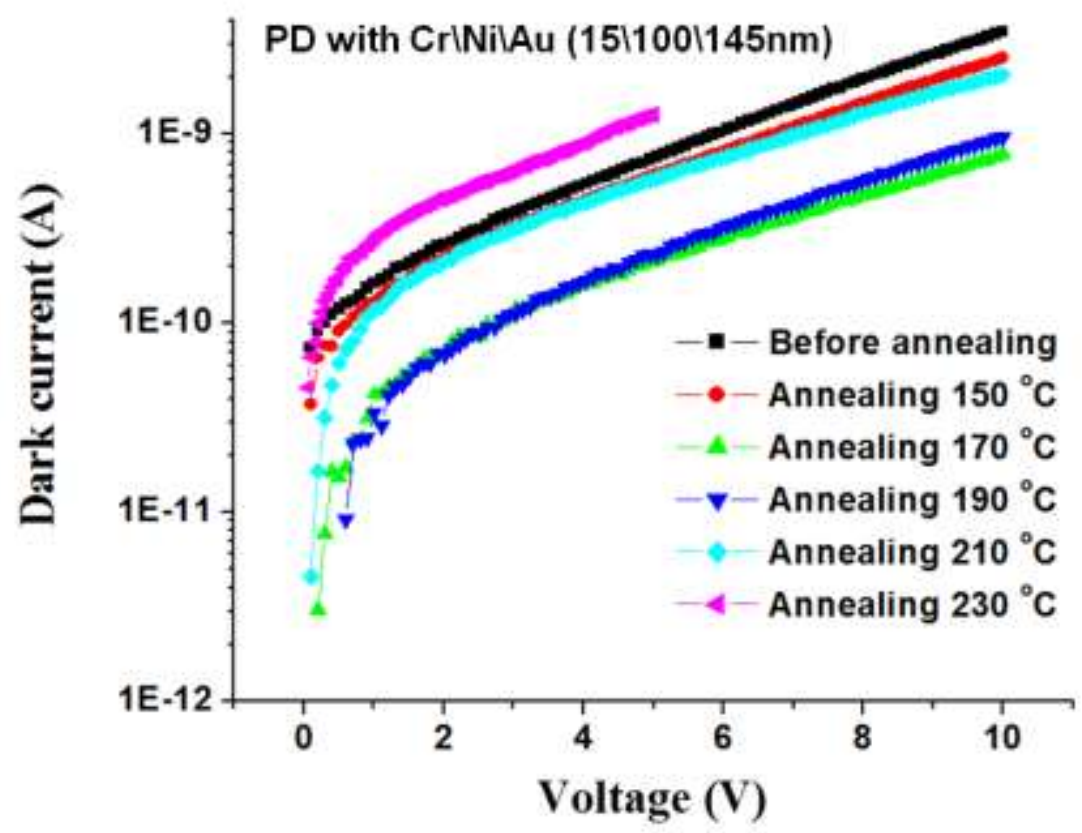

(b) 
Figure 5.

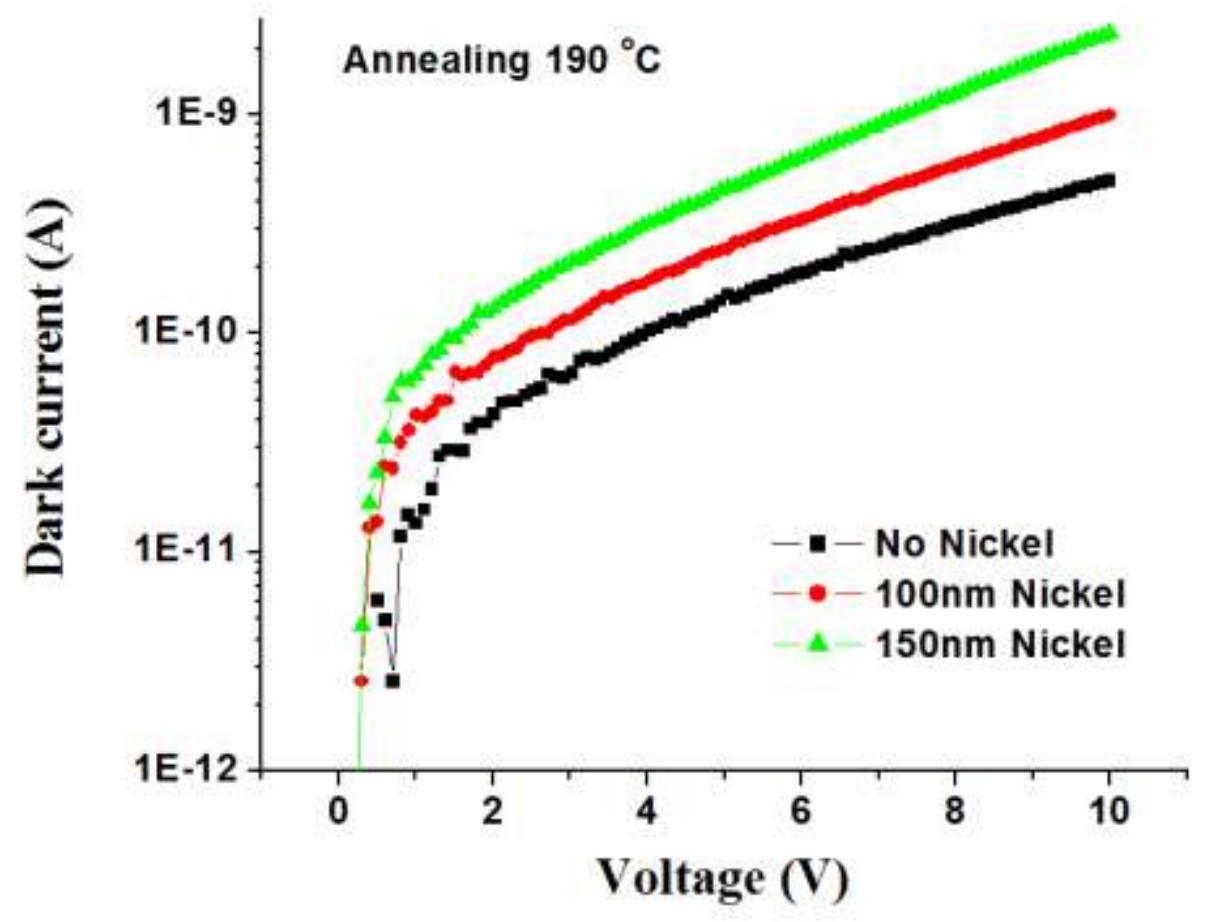


Figure 6.

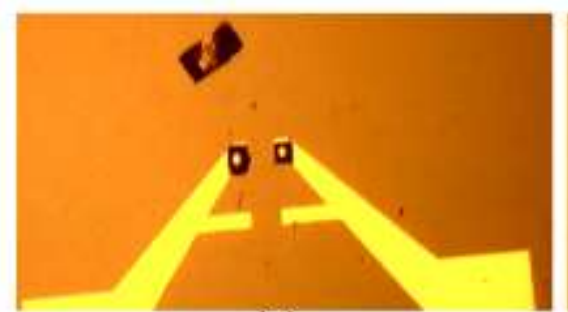

(a)

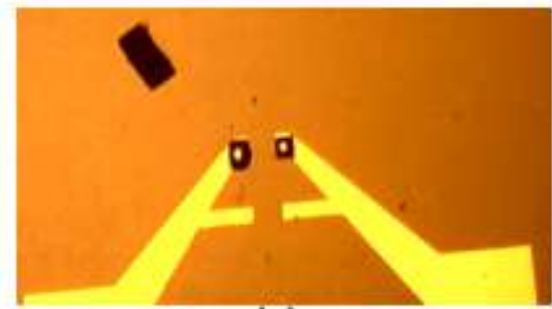

(c)

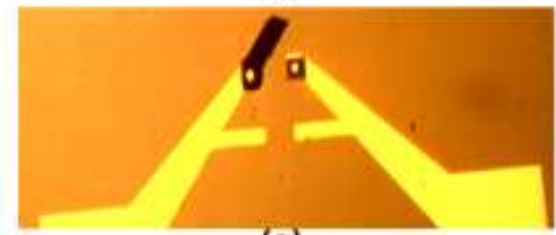

(e)

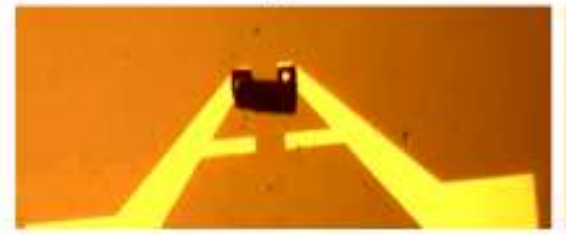

(g)

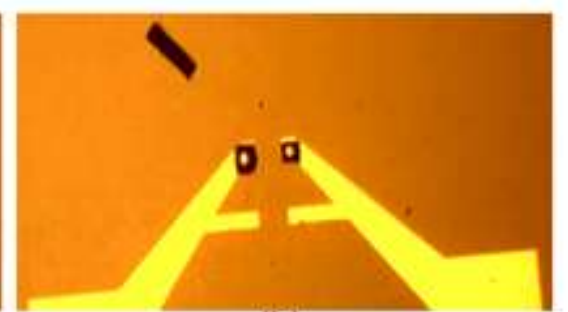

(b)

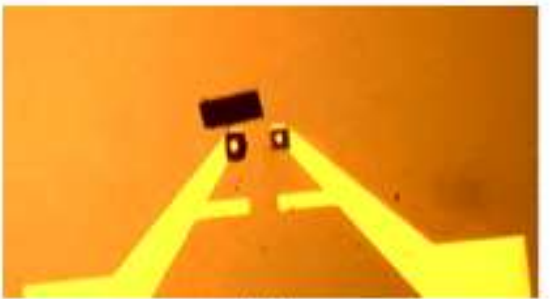

(d)

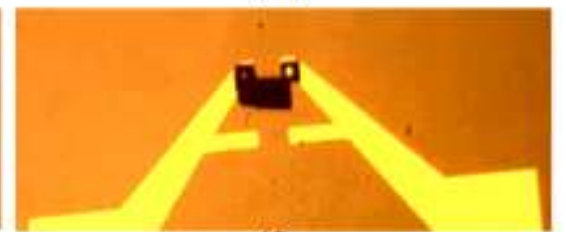

(f)

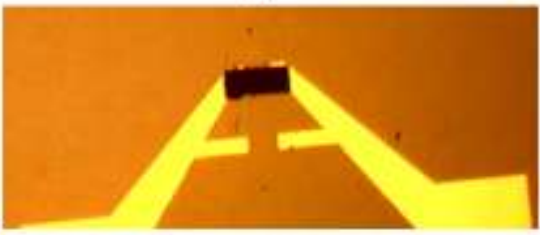

(h) 
Figure 7.

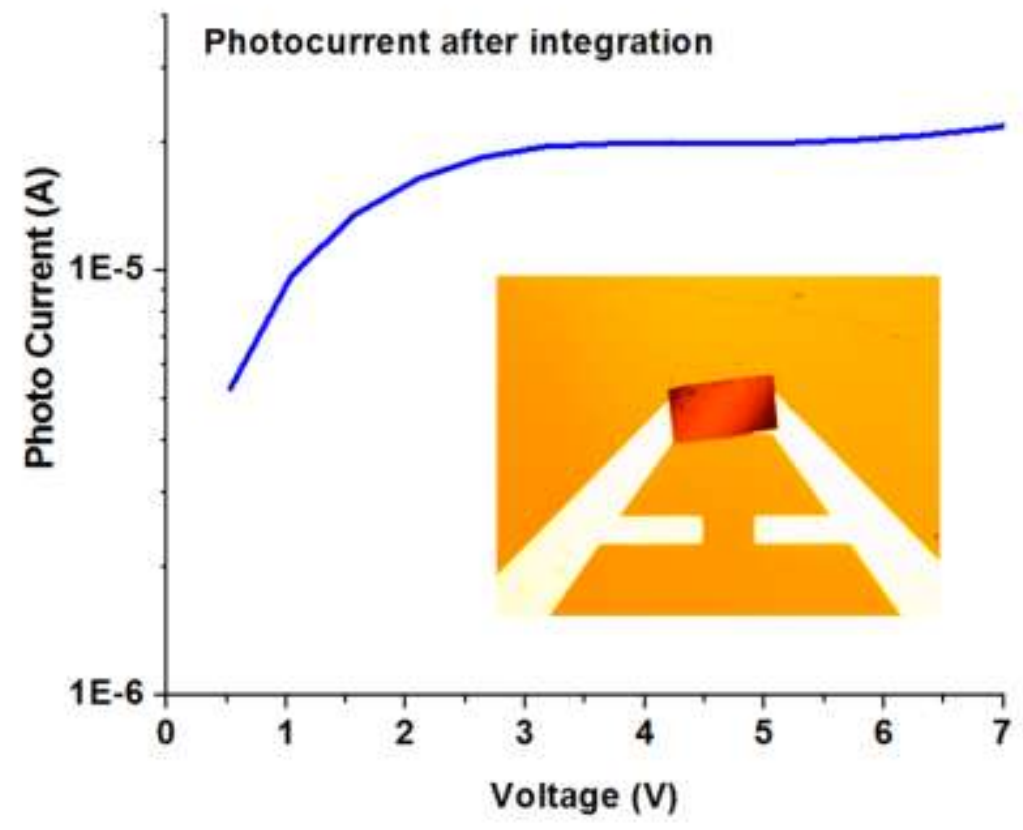


Figure 8.

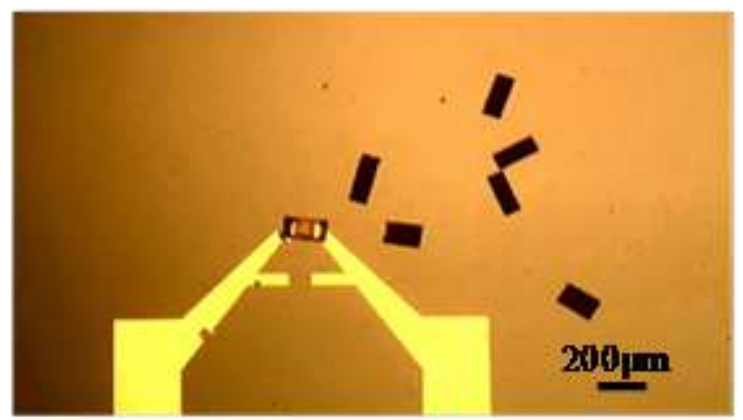

